\title{
Joule Heating in Two-dimensional Materials Based Transistors
}

ISSN: 2576-8840

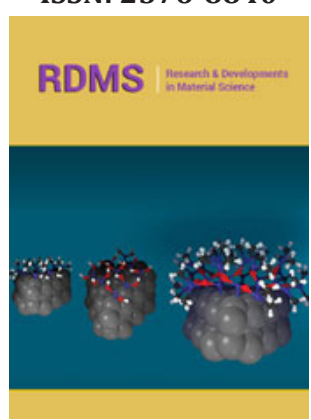

*Corresponding author: Bilal Anjum Ahmed, Department of Mechanical Engineering, College of Electrical and Mechanical Engineering, National University of Science and Technology, Islamabad-44000, Pakistan

Submission: 酒 April 01, 2019

Published: 侮 April 09, 2019

Volume 10 - Issue 4

How to cite this article: Faisal $A$, Bilal A A, Abbas S H. Joule Heating in Two-dimensional Materials Based Transistors. Res Dev Material Sci. 10(4). RDMS.000741.2019.

DOI: 10.31031/RDMS.2019.10.000741

Copyright@Bilal Anjum Ahmed, This article is distributed under the terms of the Creative Commons Attribution 4.0 International License, which permits unrestricted use and redistribution provided that the original author and source are credited.

\author{
Faisal Ahmed ${ }^{1}$, Bilal Anjum Ahmed ${ }^{1 *}$, Abbas Saeed Hakeem² \\ ${ }^{1}$ Department of Mechanical Engineering, Pakistan \\ ${ }^{2}$ Center of Excellence in Nanotechnology, Saudi Arabia
}

\begin{abstract}
Recently, two-dimensional electronic materials such as graphene, transition metal dichalcogenides and black phosphorus are persuaded as a possible alternative to the conventional silicon based electronic technology due to their exceptional characteristics. Interestingly, the two-dimensional materials exhibit drastically different electrical, mechanical, thermal and physical properties compared to their bulk counterpart materials due to the quantum confinement effect. The two-dimensional materials have been extensively studied under ambient or cryogenic operating conditions in order to explore their intrinsic characteristics and materials' physics. However, the practical electronic devices are operated under aggressive operating conditions like large electrical and thermal stresses, where heat dissipation and its management become critical. Therefore, in this report we sought to review the progress made towards Joule heating effect and remedies for efficient thermal spreading in functional two-dimensional materials-based transistors.
\end{abstract}

Keywords: Joule heating; Two-dimensional materials; Graphene; High electric field

\section{Mini Review}

Transistor is considered as a building block for electronic circuitry, as it is used to control the flow of electric current across its semiconducting channel while applying potential difference along its metallic electrodes (the source and the drain). The flow of current is regulated by a third electrode called the gate, that is separated from a semiconducting channel by an electrically insulating material, as shown schematically in Figure 1(a). When a large amount of electric field (F) [electric potential (V) normalized by channel length $(\mathrm{L}), \mathrm{F}=\mathrm{V} / \mathrm{L}$ ], is applied to a transistor, the kinetic energy of electronic charge carriers in semiconducting channel increases, resulting in the aggressive interaction of carriers with each other and with immediate environment. These charge collision events in association with atomic oscillations, also called phonons, increase the device operating temperature, and this effect is called Joule heating [1]. The extent of Joule/thermal power (P) generated depends on the electrical resistance $(R)$ of a material and the square of current (I) passed across it, as $P=I^{2} R$ [2]. In a typical integrated circuit, there are over a billion transistors that generate significant amount of thermal energy. If the Joule energy is not dissipated properly, it may lead to malfunction or eventual burning of transistors hence circuits [3].

Silicon is widely used semiconducting channel material for electronics thanks to its abundant availability, cheap and highly suitable electronic properties. Recently, there has been an aggressive push from public as well as industry for flexible, portable and highperformance electronic devices [1]. Silicon fails to meet the daunting criteria since it degrades its properties when scaled to sub-nanometer node. However, other nano materials having fascinating electrical, mechanical and thermal properties have been reported in literature [4-6]. In the emerging two-dimensional (2D) electronic materials graphene, transition metal dichalcogenides (TMDCs) and black phosphorus are considered as most suitable alternatives [2]. The naturally layered structure of these 2D materials enables them to scale down to single layer thickness that is merely $<1 \mathrm{~nm}$ in z-axis. The thinner physique of $2 \mathrm{D}$ materials means that they can easily accommodate a greater number of transistors in the vertical direction unlike bulk silicon devices thereby increasing packing density. It is pertinent to note that the greater packing density leads to the greater thermal power density in functional circuits 
based on 2D materials. In addition to this, except graphene, the semiconducting 2D materials exhibit smaller thermal conductivity values $\left(\mathrm{k}<100 \mathrm{~W} / \mathrm{m}^{2} \cdot \mathrm{K}\right)$ at room temperature, that is the bottleneck to the efficient dissipation of thermal energy [7]. To address this issue, there has been a number of reported studies. In this outlook, a systematicallyreview of Joule heating effect in 2D materials based on electronic devices.

Micro-Raman spectroscopy is widely employed to measure the localized Joule temperature in a device since Raman spectra depict the population of specific phonon modes (thermal carrier) modes of the material. Therefore, the relative position of Raman modes is considered as micro-thermometer to compute the operating temperature at a small scale [7]. In 2D materials, Joule heating was firstly reported by IBM [8]. They employed spatial Raman spectroscopy to compute the temperature distribution in functional graphene device under electrical stress. Their result showed that the center of graphene device is heated up to $1050 \mathrm{~K}$ temperature and lateral ends are relatively cooler as the metallic electrodes function as heat sink, as shown in Figure 1(b). Similarly, operating temperature of 2D semiconducting materials $\mathrm{MoS}_{2}$ and black phosphorus was also reported by micro-Raman spectroscopy [4,911]. In another study, Grosse et. al. [12] studied thermal spreading in graphene device by using thermal atomic force [12]. However, Bae et al. [13] employed thermal infrared camera to capture the heat distribution in a graphene device. However, this approach needs ultra-high-resolution IR camera to detect the thermal signals [13]. From all the reported studies it was suggested that the channeldielectric interface is the thermal bottleneck since commonly used dielectric material, $\mathrm{SiO}_{2}$, exhibit very low $\mathrm{k} \sim 1.4 \mathrm{~W} / \mathrm{m} \cdot \mathrm{K}$ at room temperature. Therefore, dielectric engineering and device structure engineering was proposed as a remedy for facile thermal spreading $[14,15]$. Graphene and black phosphorus devices fabricated on an insulating hexagonal boron nitride (hBN) substrate, showed better electrical endurance and thermal susceptibility compared to that of $\mathrm{SiO}_{2}$ supported devices due to better thermal properties of hBN $[4,16]$. Kim et al. [16] sandwiched graphene with hBN and applied a very high electrical field, in result, they observe that graphene can sustain ultra-high electrical stress and exhibited around $2800 \mathrm{~K}$ temperature [17]. Surprisingly, at very high field graphene emit visible radiations. Similarly, in another report they reported bright visible light emission from graphene by suspending it over $\mathrm{SiO}_{2}$ trench, as the thermal energy was accumulated along the center of channel, that leads to radiation emission, as shown in Figure 1(c) [18]. This was partially attributed to ultra-high $\mathrm{k}$ and very small heat capacity of graphene at room temperature. Similarly, the suspended $\mathrm{MoS}_{2}$ also exhibited similar light emission property. Ali et al. [15] proposed device structure engineering to smoothly dissipate the heat in semiconducting BP device [15].

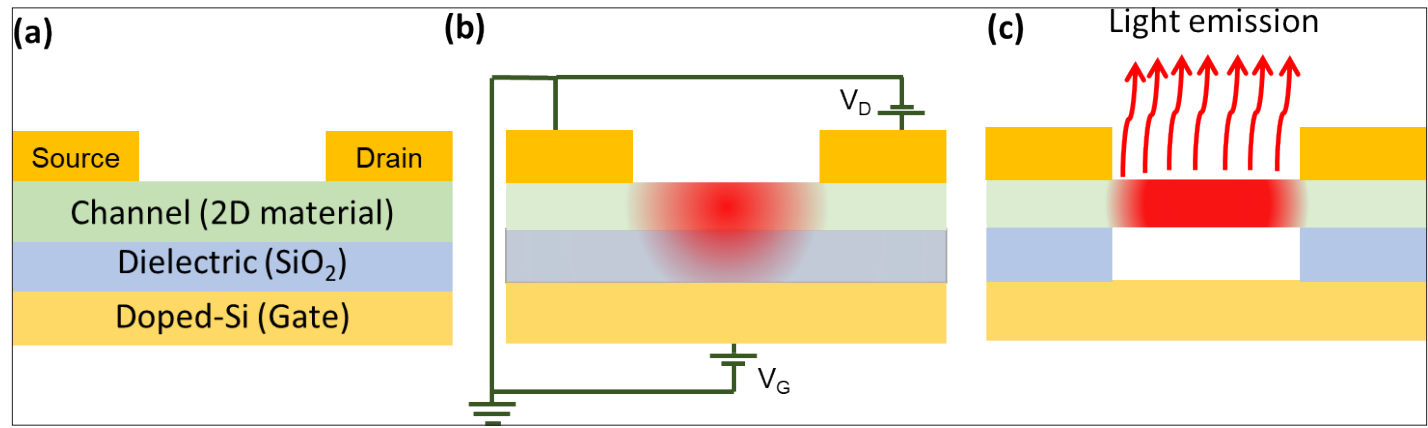

\section{Figure 1:}

(a): Schematic of a back-gate field effect transistor.

(b): Electric connection and Joule heating effect in a functional device.

(c): Schematic of light emission from the suspended device.

\section{Conclusion}

In conclusion, Joule spreading phenomenon is highly critical for practical and reliable device functionality, because Joule heating induced thermal stresses may lead to device breakdown. Although number of aforementioned studies are carried out in this area, but still, there is still ample opportunity available for future studies. For example, there is a dire need to directly measure the spatial operating temperature of the device because micro-Raman is limited to point-by-point data measurements. Furthermore, new device concepts like ballistic devices [19] and thermal rectifiers [20] are to be investigated in detail. Also, Joule heating phenomenon in many emerging 2D materials like TMDCs, hBN, transition metal carbides, nitrides and carbonitrides need to be investigated.

\section{References}

1. Song H (2018) Two-dimensional materials for thermal management applications. Joule 2(3): 442-463.

2. Lin Z (2018) Research update : Recent progress on 2D materials beyond graphene: From ripples, defects, intercalation, and valley dynamics to straintronics and power dissipation. APL Materials 6(8): 1-20.

3. Yagi M, Shirakashi J (2019) Quantifying joule heating and mass transport in metal nanowires during controlled electromigration. Materials (Basel) 12(2): 310.

4. Ahmed F (2018) Dielectric dispersion and high field response of multilayer hexagonal boron nitride. Adv Funct Mate 28(40): 1804235.

5. Irshad HM (2017) Investigation of the structural and mechanical properties of micro-/nano- sized $\mathrm{Al}_{2} \mathrm{O}_{3}$ and $\mathrm{cBN}$ composites prepared by spark plasma sintering. Ceram Int 43(14):10645-10653. 
6. Ahmed BA (2017) Effect of precursor size on the structure and mechanical properties of calcium-stabilized sialon/cubic boron nitride nanocomposites. J Alloys Compd 728: 836-843.

7. Balandin AA (2011) Thermal properties of graphene and nanostructured carbon materials. Nat Mater 10(8): 569-581.

8. Freitag M (2009) Energy dissipation in graphene field-effect transistors. Nano Lett 9(5):1883-1888.

9. Engel M, Steiner M, Han SJ, Avouris P (2015) Power dissipation and electrical breakdown in black phosphorus. Nano Lett 15(10): 67856788

10. Yalon E (2017) Energy dissipation in monolayer $\mathrm{MoS}_{2}$ electronics. Nano Lett 17(6): 3429-3433.

11. Ahmed F (2018) Impact ionization by hot carriers in a black phosphorus field effecttransistor. Nat Commun 9(1): 3414

12. Grosse KL, Bae M, Lian F, Pop E, King WP (2011) Nanoscale joule heating , Peltier cooling and current crowding at graphene - metal contacts. Nat Nanotechnol 6: 287-290.

13. Bae M, Ong Z, Estrada D, Pop E (2010) Imaging, simulation, and electrostatic control of power dissipation in graphene devices. Nano Lett 10(12): 4787-4793.
14. Ahmed F (2017) High electric field carrier transport and power dissipation in multilayer black phosphorus field effect transistor with dielectric engineering. Adv Funct Mater 27(4): 1604025.

15. Ali F (2019) Energy dissipation in black phosphorus heterostructured devices. Adv Mater Interfaces 6(2): 1801528.

16. Kim D (2018) Energy dissipation mechanism revealed by spatially resolved Raman thermometry of graphene/hexagonal boron nitride heterostructure devices. 2D Mater 5(2): 025009.

17. Kim YD (2018) Ultrafast graphene light emitters. Nano Lett 18(2): 934940.

18. Kim YD (2015) Bright visible light emission from graphene. Nat Nanotechnol 10: 676-681.

19. Sood A (2019) Quasi-ballistic thermal transport across $\mathrm{MoS}_{2}$ thin films. Nano Lett, doi:10.1021/acs.nanolett.8b05174.

20. Soo A (2018) An electrochemical thermal transistor. Nat Commun 9(1): 4510 .

For possible submissions Click below: 